\title{
Order of invasion affects the spatial distribution of a reciprocal intraguild predator
}

\author{
Tessa van der Hammen • André M. de Roos • \\ Maurice W. Sabelis · Arne Janssen
}

Received: 3 April 2009 / Accepted: 21 January 2010 / Published online: 19 February 2010

(C) The Author(s) 2010. This article is published with open access at Springerlink.com

\begin{abstract}
When intraguild predation is reciprocal, i.e. two predator species kill and feed on each other, theory predicts that well-mixed populations of the two species cannot coexist. At low levels of the shared resource, only the best competitor exists, whereas if the level of the common resource is high, the first species to arrive on a patch can reach high numbers, which prevents the invasion of the second species through intraguild predation. The order of invasion may therefore be of high importance in systems with reciprocal intraguild predation with high levels of productivity, with the species arriving first excluding the other species. However, natural systems are not well mixed and usually have a patchy structure, which gives individuals the possibility to choose patches without the other predator, thus reducing opportunities for intraguild predation. Such avoidance behaviour can cause spatial segregation between predator species, which, in turn, may weaken the intraguild interaction strength and facilitate their co-occurrence in patchy systems. Using a simple set-up, we studied the spatial distribution of two reciprocal intraguild predators when either of them was given priority on a patch with food. We released females of two predatory mite species sequentially and found that both species avoided patches on which the other species was resident. This resulted in partial spatial segregation of the species and thus a lower chance for the two species to encounter each other. Such behaviour rein-
\end{abstract}

Communicated by John Reeve.

T. van der Hammen · A. M. de Roos · M. W. Sabelis .

A. Janssen ( $\square)$

Section of Population Biology, IBED,

University of Amsterdam, Science Park 904,

1098 XH Amsterdam, The Netherlands

e-mail: arne.janssen@uva.nl forces segregation, because heterospecifics avoid patches with established populations of the other species. This may facilitate coexistence of two intraguild predators that would exclude each other in well-mixed populations.

Keywords Predator-prey interactions · Predatory mites · Antipredator behaviour · Intraguild predation · Distribution

\section{Introduction}

Intraguild predation, the killing and eating of heterospecific competitors, is common in many food webs (Polis et al. 1989; Rosenheim et al. 1995; Arim and Marquet 2004; Janssen et al. 2007, Fig. 1). However, classic theory on intraguild predation, derived from simple Lotka-Volterra models, predicts that coexistence between intraguild prey and intraguild predators is unlikely to occur frequently (Polis et al. 1989; Holt and Polis 1997; Morin 1999; Diehl and Feissel 2000; Diehl and Feissel 2001; Mylius et al. 2001). Specifically, theory predicts that coexistence is only possible with the simplest form of intraguild predation, when one competitor species (the intraguild predator) feeds on the other competitor (the intraguild prey; Fig. 1a). Even then, coexistence is predicted only when the intraguild prey is the superior competitor for the shared resource and only at intermediate levels of productivity (Polis et al. 1989; Holt and Polis 1997). At low levels of productivity, the inferior competitor for the shared resource (the intraguild predator) goes extinct through competition with the superior competitor (the intraguild prey), whereas at high levels of productivity, the intraguild predator excludes the intraguild prey due to predation (Polis et al. 1989; Holt and Polis 1997; Morin 1999; Diehl and Feissel 2000; Diehl and Feissel 2001; Mylius et al. 2001). 


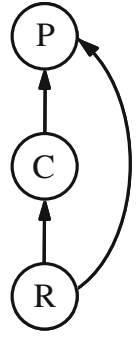

(a)

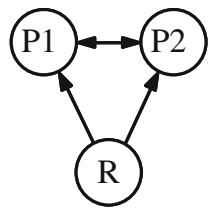

(b)

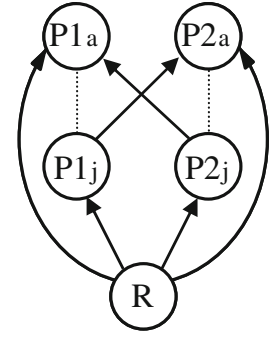

(c)
Fig. 1 Three types of intraguild predation. a Simple intraguild predation, where only one consumer feeds on the other consumer; $\mathbf{b}$ reciprocal intraguild predation, where the consumers feed on each other; $\mathbf{c}$ reciprocal intraguild predation with size structure, where both consumers feed on each other's juveniles. $R$ Resource, $C$ consumer or intraguild prey, $P$ (reciprocal) intraguild predator, $j$ juveniles, $a$ adults

Several mechanisms have been proposed to explain the discrepancy between theoretical predictions and field occurrence of intraguild predation (Holt and Polis 1997; Heithaus 2001; Snyder et al. 2005; Daugherty et al. 2007; Holt and Huxel 2007; Janssen et al. 2006, 2007; Rudolf 2007; Namba et al. 2008). Most of these proposals are based on relaxing the assumption of the general theory that systems are simple and homogeneous, and have added some type of complexity to the system. First, the basic theory on intraguild predation is restricted to only three species, whereas real systems usually consist of more species (Polis et al. 1989). Intraguild predators are omnivorous and can usually feed on more resources than only the intraguild prey and the shared resource. When alternative resources (including conspecifics in cannibalistic species) are included in the theory, the possibilities for coexistence between intraguild prey and predators are often increased (Heithaus 2001; Daugherty et al. 2007; Holt and Huxel 2007; Rudolf 2007; Namba et al. 2008). Second, theory assumes unstructured environments, whereas real systems are often heterogeneous, providing spatial or temporal refuges for prey, which may weaken the interaction between intraguild predators and intraguild prey (Finke and Denno 2006; Amarasekare 2007, Janssen et al. 2007). To minimize encounters, prey may seek refuge in structured habitats (Venzon et al. 2000; Finke and Denno 2002, 2006; Janssen et al. 2006, 2007) or may reduce their mobility (Finke and Denno 2002, 2006; Magalhaes et al. 2005b). Third, theory assumes well-mixed populations, whereas populations in real systems are distributed over several patches, which may vary in quality in space and new patches may arise through time. This gives opportunities for prey to emigrate from and avoid patches with high densities of predators. Avoidance of patches with (cues of) intraguild predators has often been observed (Moran and Hurd 1994; Faraji et al. 2000; 2001; Agarwala et al. 2003;
Sergio et al. 2003; Magalhaes et al. 2005b). This may lead to (partial) segregation of the species, which reduces the encounter rates of intraguild prey and predators and increases the possibilities for persistence of both species. Avoidance of intraguild predators is usually studied with cues of predators or with the predators unable to migrate to other patches. However, there is still a lack of research on the effect of avoidance on the distribution of intraguild species when both species are allowed to move among patches.

Another level of complexity that has been added to the basic model of intraguild predation is reciprocal intraguild predation. This occurs when two competing species consume each other (Fig. 1b, c). Even though it is observed frequently in natural systems (Polis et al. 1989), this interaction has only been investigated to a limited extent. Theory on well-mixed, homogeneous populations of reciprocal intraguild predators predicts that at high productivity levels, neither species can invade populations with high numbers of the other species, because-even when per capita intraguild predation is weak-the overall predation of the latter species imposes a high death risk for the invading species. Because of the high productivity, the competitor that arrives first can reach high numbers and thus prevents invasion of the other species. Hence, the order of invasion is expected to be of major importance in systems with reciprocal intraguild predation (HilleRisLambers and Dieckmann 2003).

In patchy systems, reciprocal intraguild predators face choices between avoidance of and attraction to patches occupied by the other predator (Faraji et al. 2001; Magalhaes et al. 2005a). Most intraguild predation is age structured or stage structured, with older or larger stages preying on younger or smaller stages of the other predator species (Mylius et al. 2001; Janssen et al. 2002; Polis et al. 1989). Thus, if intraguild predation is reciprocal, intraguild predators of both species should avoid exposure of their offspring to larger stages of the other predator, but they are also expected to be attracted towards juvenile stages of the other predator.

The first predator species to arrive on a patch will have the opportunity to build up a population, thereby deterring the other species. If both species succeed in occupying one or several patches, this could result in avoidance of these patches by the other species and thus lead to segregation. Thus, we expect an effect of the order of invasion of the two species on the distribution of intraguild predators.

We tested the hypothesis that the prior establishment of a reciprocal intraguild predator on a patch with food causes another reciprocal intraguild predator to avoid these patches and instead to occupy an alternative patch with food. More specifically, we studied whether two predatory mites (Iphiseius degenerans and Neoseiulus cucumeris), which are known to feed on each other's juveniles and 
compete for alternative food sources such as thrips, larvae and pollen (Van Rijn and Tanigoshi 1999), avoid patches with high densities of the other species.

\section{Materials and methods}

\section{Cultures}

The predatory mites Iphiseius degenerans (Berlese) and Neoseiulus cucumeris (Oudemans) were cultured in a climate room at $25 \pm 1{ }^{\circ} \mathrm{C}, 16: 8 \mathrm{~h}$ light:dark, and $60 \pm 5 \%$ relative humidity $(\mathrm{RH})$. Mites were held on plastic arenas $(30 \times 21 \mathrm{~cm})$ placed on top of sponges in water-containing trays. Small threads were added to the arenas, serving as oviposition sites. Twice per week, $N$. cucumeris was fed cattail pollen (Typha ssp.) and I. degenerans was fed birch pollen (Betula ssp). Every week, eggs were collected from the cultures and placed on new plastic arenas to obtain cohorts of mites of similar age. For more details on culture methods see Van Rijn and Tanigoshi (1999). Experiments were carried out in a climate room at $25 \pm 1{ }^{\circ} \mathrm{C}, 16 \mathrm{~h}$ light per day, and $60 \pm 5 \%$ RH. All mites used in the experiments were adult females, aged between 10 and 15 days, corresponding to the age at which food intake and oviposition rates are highest (Van Rijn and Tanigoshi 1999).

\section{Experimental set-up}

The experimental set-up consisted of five plastic patches ( $3 \mathrm{~cm}$ diameter) in a circle, each patch being connected to the two nearest patches through a small plastic bridge (Fig. 2). We added ample amounts (approximately $3 \mathrm{mg}$ ) of food (Typha ssp. pollen) to two patches [pollen only (patch $\mathrm{P}$ ) and resident (patch R)]; three patches remained without food [invader (patch $\mathrm{I}_{\mathrm{nv}}$ ) and two empty, no-pollen patches (E)]. The patch $\mathrm{I}_{\mathrm{nv}}$ was used as a release patch (see "Introduction of mites") and was situated in between the two patches with pollen. The two E patches (Fig. 2) were added in order to detect possible avoidance of ovipositing on the patches with food, which were expected to contain high numbers of mites. The entire arena was cut out from a single sheet of plastic. A piece of thread (approximately $1.5 \mathrm{~cm}$ ) was glued to each patch to serve as a substrate for oviposition. This provided ample space for oviposition throughout the experiments. The circle of patches was placed on wet cotton wool, which prevented the mites from leaving the arena and served as a water source for the mites.

\section{Introduction of mites}

We tested the effect of the order of invasion of the two mite species by allowing 15 adult females of one of the two spe-

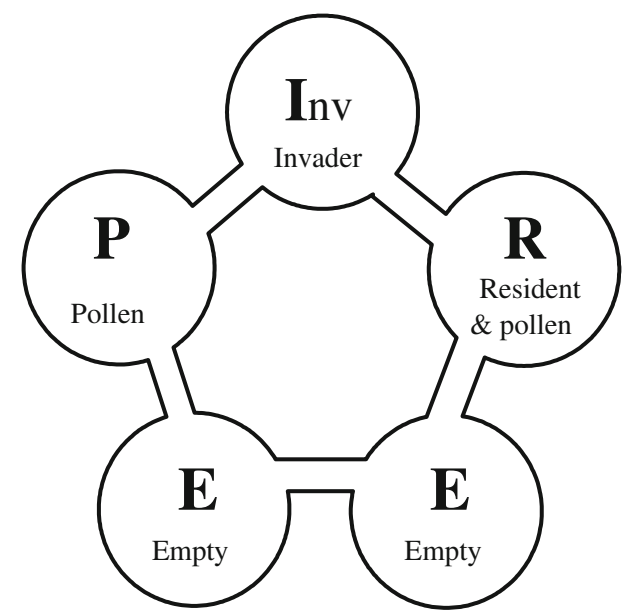

Fig. 2 The experimental set-up. Predatory mites of two different species were released either $23 \mathrm{~h}$ prior to the start of the experiment on the resident patch $(R)$, which was supplied with ample pollen as food, or $1 \mathrm{~h}$ prior to the start of the experiment on the invader patch $\left(I_{\mathrm{nv}}\right)$, without pollen. No mites were released on the ample pollen patch $(P)$, or the empty, no-pollen patches $(E)$. All patches had a small piece of thread, which the mites used for oviposition

cies to settle on a patch with pollen. We will refer to these mites as the "resident species" and to the patch on which they were released as the "resident patch" (i.e. patch R; Fig. 2). Barriers of wet cotton wool were put over the adjacent bridges to confine the mites to the patch. Twenty-three hours later, 15 adult females of the other species were released on the empty patch that was located in between the two pollen patches at equal distance $\left(\mathrm{I}_{\mathrm{nv}}\right.$; Fig. 2$)$. These mites were confined there by similar barriers on bridges for a maximum of $1 \mathrm{~h}$ and they are referred to as the "invading species". Twenty-four hours after the release of the resident species, the eggs on the resident patch were counted. There were no eggs at patch $\mathrm{I}_{\mathrm{nv}}$, because the mites were confined only for a short period on this patch. Subsequently, all barriers were removed, and the individuals on the invasion patch could then choose to move to a food patch that was predominantly occupied with intraguild predators, or to the patch with pollen without the competing predator. The resident predators were also free to move from their patch to other patches. The two food patches were considered of equal quality because food was amply present on both patches throughout the entire experiment, and was by no means depleted on the resident patch. Control treatments consisted of 15 I. degenerans or $15 \mathrm{~N}$. cucumeris alone on either the patch on which the invading species was released $\left(\mathrm{I}_{\mathrm{nv}}\right)$ or on the pollen patch $\mathrm{R}$, where they received the same treatment as above. Hence, they were confined to patch $\mathrm{R}$ for $24 \mathrm{~h}$ or to patch $\mathrm{I}_{\mathrm{nv}}$ for $1 \mathrm{~h}$. We will also refer to these populations as "resident" when released on patch $\mathrm{R}$, and "invading" when released on patch $\mathrm{I}_{\mathrm{nv}}$, although the invading mites in this case enter into an environment without the other species present. The number of replicates was 14 or 15 in all cases. 
We selected an additive design because we think it is the best way to study the influence of one species on another. We realize that effects could also be partly due to density differences, but there is no proper control for densities of heterospecifics, since this would be based on the assumption that an individual of one species causes the same density effect as an individual of another species (Jolliffe 2000). However, we have observed that oviposition by $N$. cucumeris was not affected by conspecific densities when there was no competition for food, whereas I. degenerans slightly decreased its oviposition when the density of conspecifics increased, even with an ample supply of food (Van der Hammen et al., in preparation). After removal of the barriers, the position and number of all mites was scored at 5, 15, and $30 \mathrm{~min}, 1,2,3$, and $24 \mathrm{~h}$, resulting in time series of the distributions.

Adults of both species can be readily distinguished by their colour. Eggs of I. degenerans and N. cucumeris can be distinguished by their size $\{N$. cucumeris, $0.21 \mathrm{~mm} \pm$ $0.002 \times 0.13 \mathrm{~mm} \pm 0.002 \mathrm{~mm}$ [length $(l) \times$ width $(w)$ ] I. degenerans $0.24 \mathrm{~mm} \pm 0.003 \times 0.18 \mathrm{~mm} \pm 0.003(1 \times \mathrm{w})$, cut-off egg size $=0.155 \mathrm{~mm}$ width $\}$. They were counted only at the end of the experiment because they are laid in clusters, and could therefore not be counted during the experiment without disturbing the mites and thus affecting their distribution. All experiments were performed at $25^{\circ} \mathrm{C}$, $16 \mathrm{~h}$ light, and $65 \% \mathrm{RH}$. Note that the two mite species do not attack and kill eggs or adults of the other species (Faraji et al. 2000; Montserrat et al. 2007); hence, no intraguild predation occurred in the experiments.

Statistical analysis

\section{Distribution of mites and eggs}

The number of mites was fixed per arena, thus the number of mites on each patch was dependent on the number of mites on the other patches. Generalized models allow for binomial error distributions, but not for multinomial distribution. Because most replicates had only small proportions of mites residing on the three patches without food (patches $\mathrm{I}_{\mathrm{nv}}$ and E; Fig. 2), we summed these proportions and we performed two regression analyses with binomial errors. First we analysed if there were differences in the distribution of mites between the summed proportion on the three patches without food and the summed proportion on the two patches with food (i.e. $\mathrm{I}_{n v}+\mathrm{E}$ vs. $\mathrm{P}+\mathrm{R}$ ). Second, we tested for effects on the proportion of mites on the pollen patches ( $\mathrm{P}$ vs. $\mathrm{R}$ ) only, ignoring the proportion of mites that were on the other three patches. If the first test was significant, this would mean that the presence of mites of the other species resulted in a different total proportion of mites on the two patches with food. If the second test was signifi- cant, this would mean that the presence of mites of the other species caused a different distribution of the first mite species over the two patches with food ( $\mathrm{R}$ and $\mathrm{P}$ ).

For the analysis of the time series, we used generalized linear mixed effect models with a binomial error distribution (GLMM; function lmer of the library lme4 in R) with treatment (presence or absence of the other species) and time as fixed effects and replicate as random effect to correct for repeated measurement. The data were only slightly overdispersed, allowing us to use a binomial error distribution. The distributions of mites and eggs in the last time step (after $24 \mathrm{~h}$ ) were also tested separately, because we expected the mites to need some time before settling on a patch. Similar to the time series analysis, we performed two tests, but because these were not repeated measures, we used generalized linear models (GLM), with treatment (presence or absence of the other species) as categorical factor. As the data of the distribution of the mites were only slightly overdispersed, we used binomial error distributions. The data of the distribution of the eggs, however, were often highly overdispersed, and we therefore used quasi-binomial error distributions (Crawley 2007).

\section{Total number of eggs}

Differences in the total number of eggs laid per species were analysed using GLM with Poisson errors (there was no serious overdispersion). During the experiments, few mites tried to escape and ended up in the water. Differences in the number of escapes were also tested using GLM with Poisson errors (no overdispersion). All statistics were performed in $\mathrm{R}$ version 2.8.1 ( $\mathrm{R}$ Development Core Team 2006).

\section{Results}

I. degenerans resident, $N$. cucumeris invading: response of N. cucumeris

\section{Distribution through time (Fig. 3a, b)}

Distribution over patches with and without food: The distribution of invading adult $N$. cucumeris over the pollen patches and the empty patches $\left(\mathrm{I}_{\mathrm{nv}}+\mathrm{E}\right.$ vs. $\left.\mathrm{P}+\mathrm{R}\right)$ through time differed significantly with the presence or absence of I. degenerans as the resident species. The proportion of $N$. cucumeris adults on the patches with pollen $(\mathrm{P}+\mathrm{R})$ was lower in the presence than in the absence of I. degenerans (GLMM, binomial, $\mathrm{I}_{\mathrm{nv}}+\mathrm{E}$ vs. $\mathrm{P}+\mathrm{R}, \chi_{1}^{2}=8.95, P=0.003$ ). In addition, the interaction of treatment with time was also significant (GLMM, binomial, treatment $\times$ time, 
Fig. 3a-h Time series of the distribution of mites on the patches with food, $\mathrm{P}$ and $\mathrm{R}$ (Fig. 2). The resident was released $24 \mathrm{~h}$ before the start of the experiment on patch $\mathrm{R}$, which had ample food. There was also ample food on patch $\mathrm{P}$, on which no mites were released. Shown are the proportions of mites on the two food patches R and P. Note that these fractions do not necessarily sum to 1 , because a proportion of the mites resided at the patches without food (see Fig. 2). a, c, e, $\mathbf{g}$ Time series in the treatments with two species; $\mathbf{b}, \mathbf{d}, \mathbf{f}, \mathbf{h}$ corresponding controls with only one species. a-d Response of Neoseiulus cucumeris, $\mathbf{e}-\mathbf{h}$ response of Iphiseius degenerans. For abbreviations, see Fig. 2
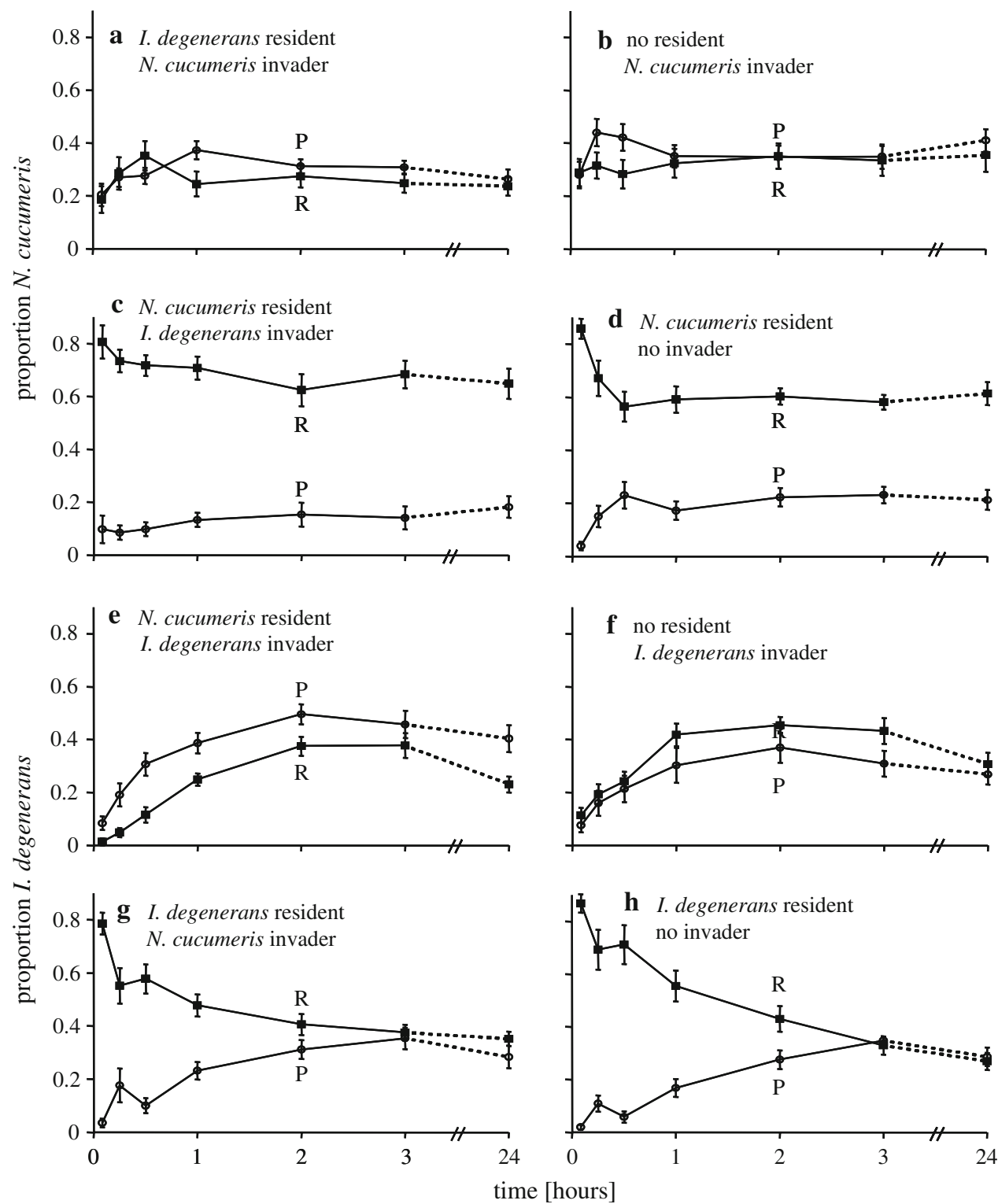

$\chi_{1}^{2}=8.18, P=0.004$ ), thus the difference in distribution between treatments changed over time. There was no effect of time, thus the same proportion of $N$. cucumeris remained on the pollen patches during the experiment (GLMM, binomial, $\chi_{1}^{2}=0.80, P=0.37$ ).

Distribution over food patches: The distribution of $N$. cucumeris over the patches with pollen (P vs. R) was not significantly affected by the presence of I. degenerans (GLMM, binomial, $\chi_{1}^{2}=0.0001, P=0.99$ ). Likewise, neither time nor the interaction of treatment with time was significant (GLMM, binomial, treatment $\times$ time, $\chi_{1}^{2}=000001, P=0.99$; time, $\chi_{1}^{2}=0.046, P=0.83$ ). Thus, $N$. cucumeris did not discriminate between the patch where I. degenerans was resident $(\mathrm{R})$ and the alternative pollen patch $(\mathrm{P})$.

Final distribution (Fig. 4a): After 24 h, the differences in the distribution were similar to those throughout the time series. The proportion of N. cucumeris on the patches with pollen ( $\mathrm{P}$ and $\mathrm{R})$ was still lower in the presence of I. degenerans than in its absence (GLM, binomial, $\mathrm{I}_{\mathrm{nv}}+\mathrm{E}$ vs. $\left.\mathrm{P}+\mathrm{R}, \chi_{1}^{2}=31.7, P<0.001\right)$. There was no difference in distribution of mites over the patches with pollen between the treatments with and without I. degenerans (GLM, binomial, P vs. R, $\chi_{1}^{2}=0.059, P=0.81$ ).

Distribution of eggs (Fig. 5a): The proportion of eggs of $N$. cucumeris on the pollen patches after $24 \mathrm{~h}$ was significantly lower in treatments with I. degenerans as resident (GLM, quasi-binomial, $\mathrm{I}_{\mathrm{nv}}+\mathrm{E}$ vs. $\mathrm{P}+\mathrm{R}, F_{1,28}=13.80$, $P<0.001)$. The distribution over the two pollen patches did not differ significantly (GLM, quasi-binomial, $\mathrm{P}$ vs. R, $\left.F_{1,28}=1.78, P=0.19\right)$. Thus, $N$. cucumeris avoided ovipositing on both pollen patches in the presence of I. degenerans. Note that the data of the distribution of the eggs were overdispersed; we therefore used quasi-binomial 
Fig. 4a-d Distribution of mites after $24 \mathrm{~h}$ on the patches with food, P and R (see Fig. 2). These are the same data as in Fig. 3 at $t=24 \mathrm{~h}$. Shown are the proportions of mites on the two food patches, R and P. a, d

I. degenerans was the resident (released on patch R),

$N$. cucumeris the invader (released on patch $\mathrm{I}_{\mathrm{nv}}$; Fig. 2). b, c $N$. cucumeris was the resident (patch R) and I. degenerans the invader $\left(\right.$ patch $\left.\mathrm{I}_{\mathrm{nv}}\right)$. *** indicates that the distribution over the summed pollen patches $(\mathrm{P}+\mathrm{R})$ versus the summed empty patches $($ Inv + E) differed between treatments $(P<0.001) .{ }^{\#}$ indicates that the distribution over the two pollen patches (P vs. R) differed between treatments $(P<$ $0.01)$. For abbreviations, see

Fig. 2
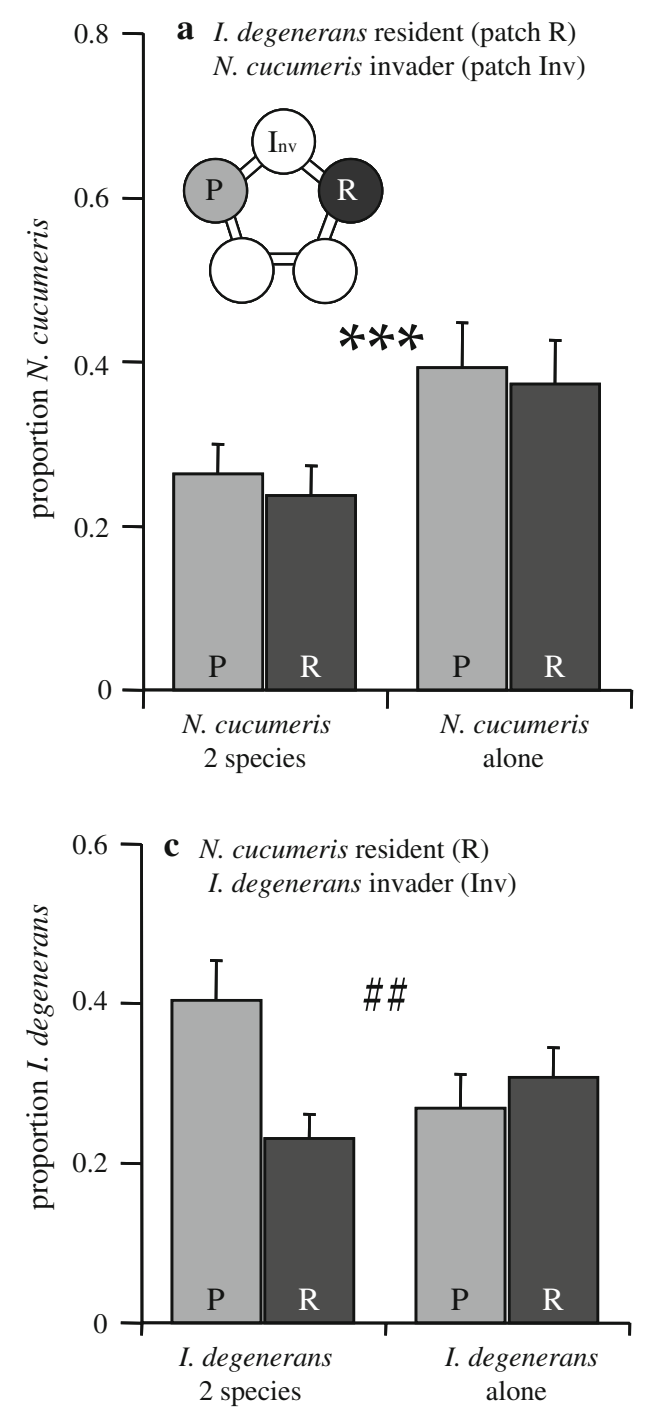

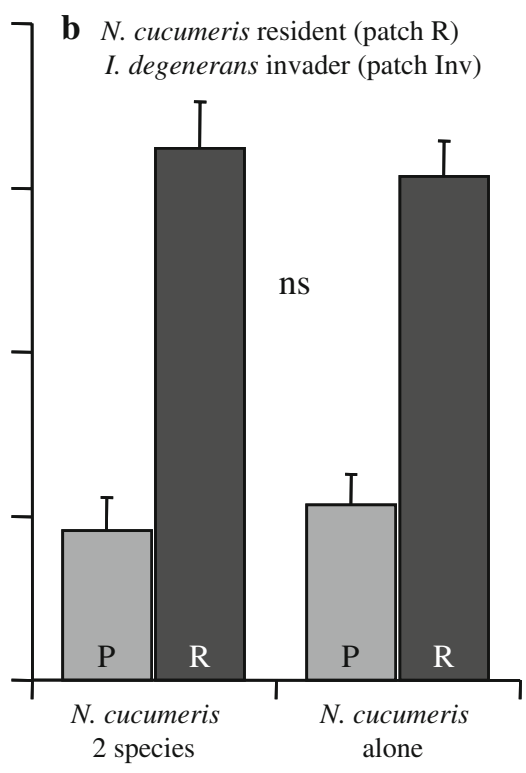

d I. degenerans resident (R) N. cucumeris invader (Inv)

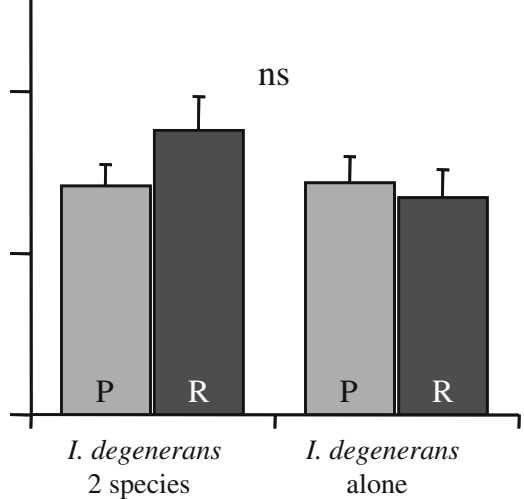

error distributions and $F$ tests instead of $\chi^{2}$ test (Crawley 2007).

Total number of eggs and escaped mites (Figs. 6a, 7a): The total number of N. cucumeris eggs (summed over all patches) was lower with I. degenerans as resident than in the absence of I. degenerans (GLM, Poisson, $\chi_{1}^{2}=26.8$, $P<0.001)$. N. cucumeris also attempted to escape more from the arena in the presence of I. degenerans (GLM, Poisson, $\chi_{1}^{2}=18.6, P<0.001$ ).

\section{I. degenerans resident, $N$. cucumeris invading: response of $N$. cucumeris}

\section{Distribution through time (Fig. 3c, d)}

Distribution over patches with and without food: A large proportion of adult $N$. cucumeris remained on the resident patch, irrespective of the presence of invading
I. degenerans. As expected, the proportion of N. cucumeris on the pollen patches did not differ between treatments with I. degenerans present or absent when N. cucumeris was the resident species $\left(\mathrm{I}_{\mathrm{nv}}+\mathrm{E}\right.$ vs. $\mathrm{P}+\mathrm{R}$, GLMM, binomial, $\left.\chi_{1}^{2}=0.17, P=0.68\right)$. There was no significant interaction of treatment with time (GLMM, binomial, $\chi_{1}^{2}=0.11$, $P=0.74$ ), or a significant effect of time (GLMM, binomial, $\chi_{1}^{2}=0.03, P=0.87$ ). Thus, the proportion of $N$. cucumeris on the pollen patches did not change over time.

Distribution over food patches: The distribution of mites over the two pollen patches ( $\mathrm{P}$ vs. $\mathrm{R}$ ) did not differ significantly between the two treatments, although there was a trend of more $N$. cucumeris residing on the resident patch in the presence of I. degenerans (GLMM, binomial, $\chi_{1}^{2}=3.17$, $P=0.075)$. The interaction of treatment with time was not significant either (GLMM, binomial, $\chi_{1}^{2}=1.27, P=0.26$ ), but time was highly significant (GLMM, binomial, time $\times$ treatment, $\chi_{1}^{2}=12.4, P<0.001$ ). 
Fig. 5a-d Distribution of eggs after $24 \mathrm{~h}$ on the patches with food, P and R. Shown are the proportions of eggs on the two food patches R and P. a, d I. degenerans was the resident (released on patch R; Fig. 2), $N$. cucumeris the invader (released on patch $\mathrm{I}_{\text {nv }} ;$ Fig. 2). b, c $N$. cucumeris was the resident (released on patch R),

I. degenerans the invader (released on patch $\mathrm{I}_{\mathrm{nv}}$ ). *** indicates that the distribution (pollen patches vs. empty patches) in the treatment with both species differs significantly from that with one species $(P<0.001)$. Ns not significant. For abbreviations, see Fig. 2
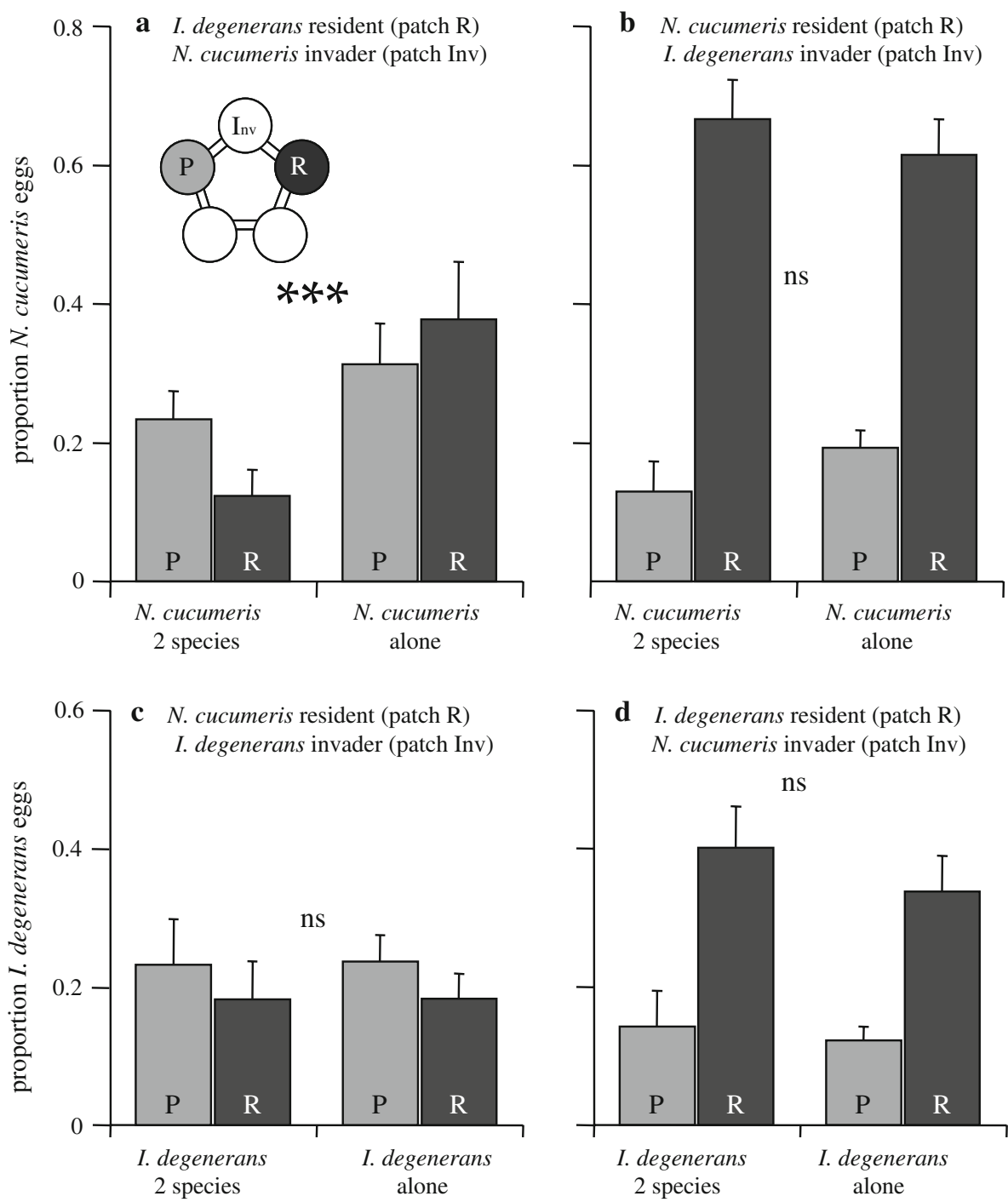

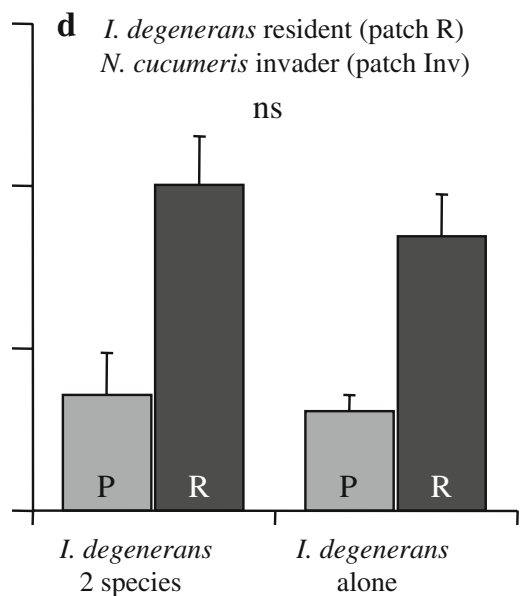

Final distribution (Fig. 4b): After $24 \mathrm{~h}$, the distribution of $N$. cucumeris did not differ between treatments with or without I. degenerans. The distribution of mites over the pollen patches and the empty patches did not differ (GLM, binomial, $\mathrm{I}_{\mathrm{nv}}+\mathrm{E}$ vs. $\mathrm{P}+\mathrm{R}, \chi_{1}^{2}=0.74, P=0.39$ ), neither did the distribution over the two pollen patches (GLM, binomial, $\mathrm{P}$ vs. $\left.\mathrm{R}, \chi_{1}^{2}=0.02, P=0.90\right)$. Thus, when $N$. cucumeris was the resident species, its distribution was not affected by invading I. degenerans.

Distribution of eggs (Fig. 5b): The distribution of eggs of $N$. cucumeris did not differ between treatments with or without invading I. degenerans (GLM, quasi-binomial, $\mathrm{I}_{\mathrm{nv}}+\mathrm{E}$ vs. $\mathrm{P}+\mathrm{R}, \quad F_{1,28}=1.59, \quad P=0.22 ; \quad \mathrm{P}$ vs. R, $\left.F_{1,28}=0.014, P=0.91\right)$.

Total number of eggs and escaped mites (Figs. 6b, 7b): The total number of eggs did not differ between treatments with or without invading I. degenerans (GLM, Poisson, $\chi_{1,28}^{2}=0.21, P=0.65$ ). There were more $N$. cucumeris escaping with invading I. degenerans compared to the treatment without invaders (GLM, Poisson, $\chi_{1}^{2}=3.3$, $P=0.070$ ), but this was a non-significant trend. Thus, the distribution of resident $N$. cucumeris was not affected by the presence of invading I. degenerans.

$N$. cucumeris resident, I. degenerans invading: response of I. degenerans

\section{Distribution through time (Fig. 3e, f)}

Distribution over patches with and without food: The proportion of invading I. degenerans on the food patches $(\mathrm{P}+\mathrm{R})$ compared to the empty patches $\left(\mathrm{I}_{\mathrm{nv}}+\mathrm{E}\right)$ did not differ between the treatments with and without resident $N$. cucumeris (GLMM, binomial, pollen patches vs. empty patches, $\left.\chi_{1}^{2}=0.0014, P=0.97\right)$. The interaction of treatment with time was nearly significant (GLMM, binomial, $\left.\chi_{1}^{2}=3.71, P=0.054\right)$. Time did have a large effect on the distribution of $I$. degenerans (GLMM, binomial, $\chi_{1}^{2}=33.9$, 
Fig. 6a-d Average numbers of eggs laid per species per treatment (+SE). Grey bars Both species present, black bars only one species present. a, $\mathbf{d}$ I. degenerans was the resident (released on patch R),

$N$. cucumeris the invader (released on patch $\mathrm{I}_{\mathrm{nv}} ;$ Fig. 2). b, c N. cucumeris was the resident (released on patch R), I. degenerans the invader (released on patch $\mathrm{I}_{\mathrm{nv}}$ ). Symbols indicate that the number of eggs differed between treatments (*** $P<0.001$, ** $P<0.01$, $n s$ not significant). For abbreviations, see Fig. 2
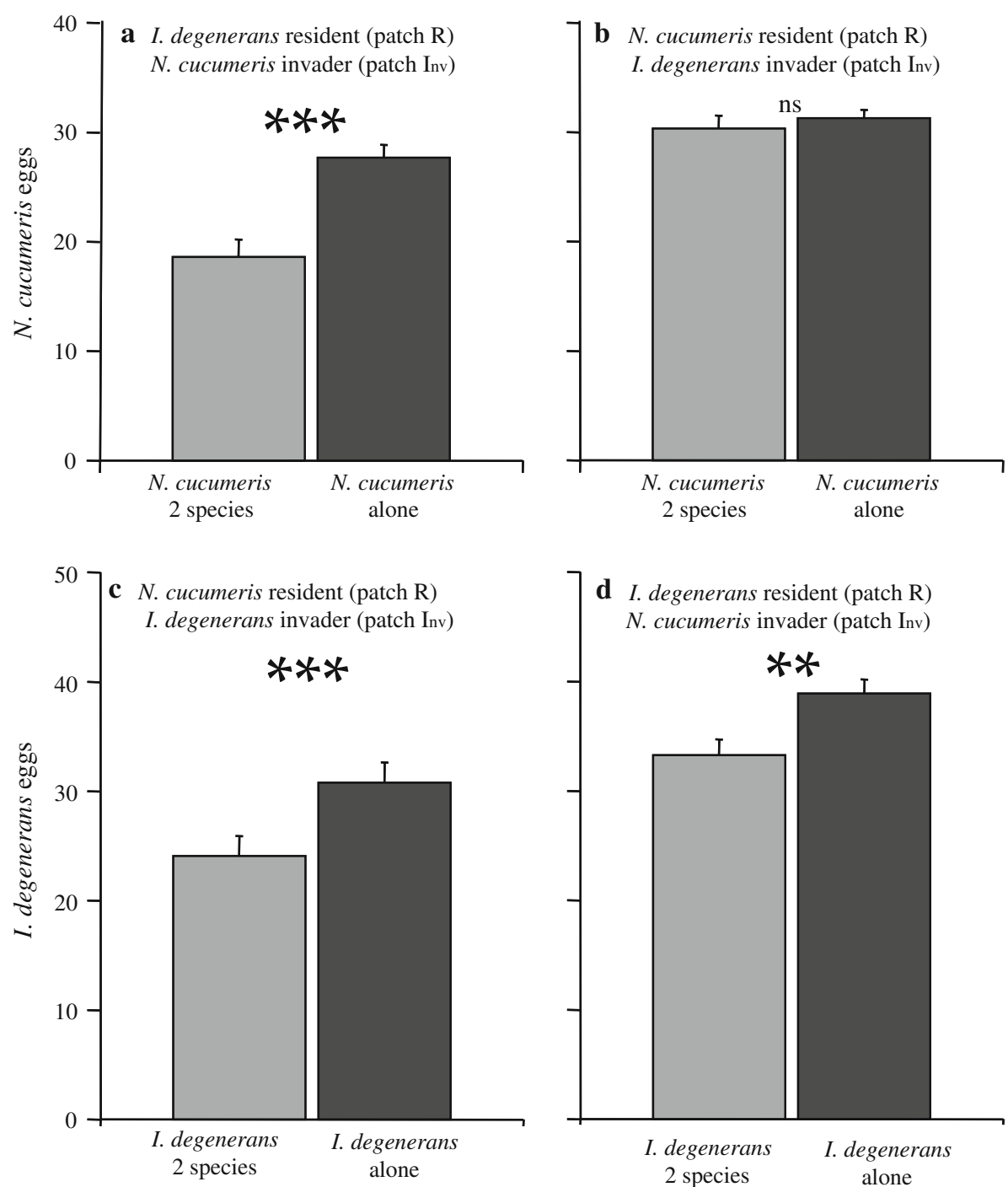

$P<0.001)$. Thus, the distribution of $I$. degenerans over the pollen patches and the empty patches changed through time but this change did not differ between treatments with or without $N$. cucumeris as resident.

Distribution over food patches: The distribution over the two patches with food (P vs. R) also differed (GLMM, binomial, $\mathrm{P}$ vs. R, $\chi_{1}^{2}=10.83, P<0.001$ ); a higher proportion of $I$. degenerans chose the empty food patch $\mathrm{P}$ instead of the alternative patch $\mathrm{R}$ in the presence of $N$. cucumeris than in its absence. The distribution of I. degenerans over the pollen patches did not change with time (GLMM, binomial, treatment $\times$ time, $\quad \chi_{1}^{2}=0.50, \quad P=0.48$; time, $\left.\chi_{1}^{2}=0.48, P=0.49\right)$.

Final distribution (Fig. 4c): After 24 h, the total proportion of mites on the patches with pollen did not differ between treatments (GLM, $\mathrm{I}_{\mathrm{nv}}+\mathrm{E}$ vs. $\mathrm{P}+\mathrm{R}$, binomial, $\chi_{1}^{2}=1.52, P=0.22$ ), whereas the distribution over the food patches still differed significantly between treatments (GLM, binomial, $\mathrm{P}$ vs. $\mathrm{R}, \chi_{1}^{2}=7.88, P=0.005$ ). Thus, I. degenerans avoided settling on the patch with resident N. cucumeris.

Distribution of eggs (Fig. 5c): The distribution of I. degenerans eggs did not differ in the presence or absence of resident $N$. cucumeris (GLM, quasi-binomial, $\mathrm{I}_{\mathrm{nv}}+\mathrm{E}$ vs. $\mathrm{P}+\mathrm{R}, \quad F_{1,28}=0.54, \quad P=0.82 ; \quad \mathrm{P}$ vs. $\mathrm{R}, \quad F_{1,28}=0.31$, $P=0.58)$. Thus, $I$. degenerans avoided patches with many $N$. cucumeris, but did not avoid laying eggs on the patches with many $N$. cucumeris.

Total number of eggs and escaped mites (Figs. 6c, 7c): The total number of eggs of I. degenerans was significantly lower in the presence of $N$. cucumeris than in their absence (GLM, Poisson, $\chi_{1}^{2}=12.0, \quad P<0.001$ ). The number of escaped mites did not differ between treatments (GLM, Poisson, $\left.\chi_{1}^{2}=0.14, P=0.71\right)$. 
Fig. 7a-d Average numbers of escapes per treatment (+SE). Grey bars Both species present, black bars one species present. a, $\mathbf{d}$ I. degenerans was the resident (released on patch R), $N$. cucumeris the invader (released on patch $\mathrm{I}_{\mathrm{nv}}$; Fig. 2). b, c $N$. cucumeris was the resident, I. degenerans the invader. *** the number of escapes differed significantly between treatments $(P<0.001$. Ns not signficant $)$. For abbreviations, see Fig. 2

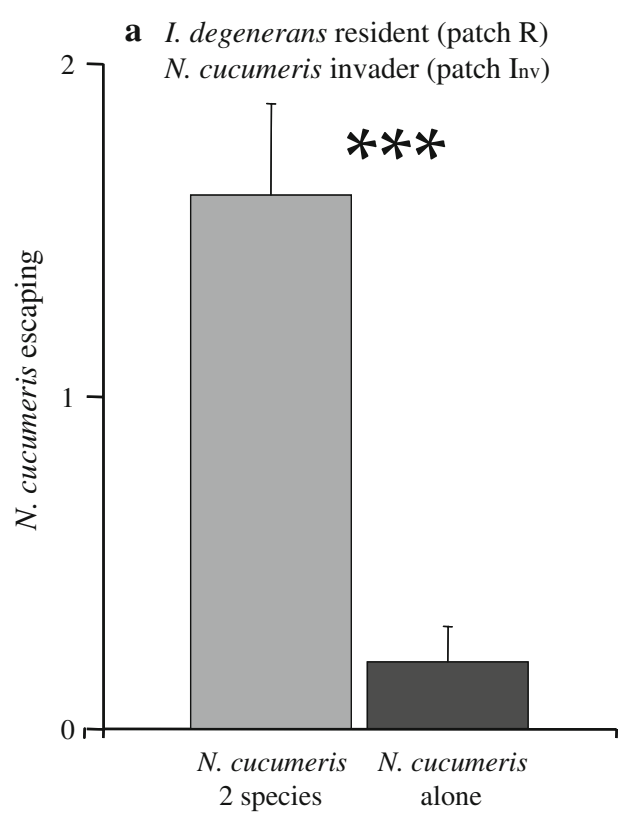

b $\quad$. cucumeris resident (patch $\mathrm{R}$ )

I. degenerans invader (patch Inv)
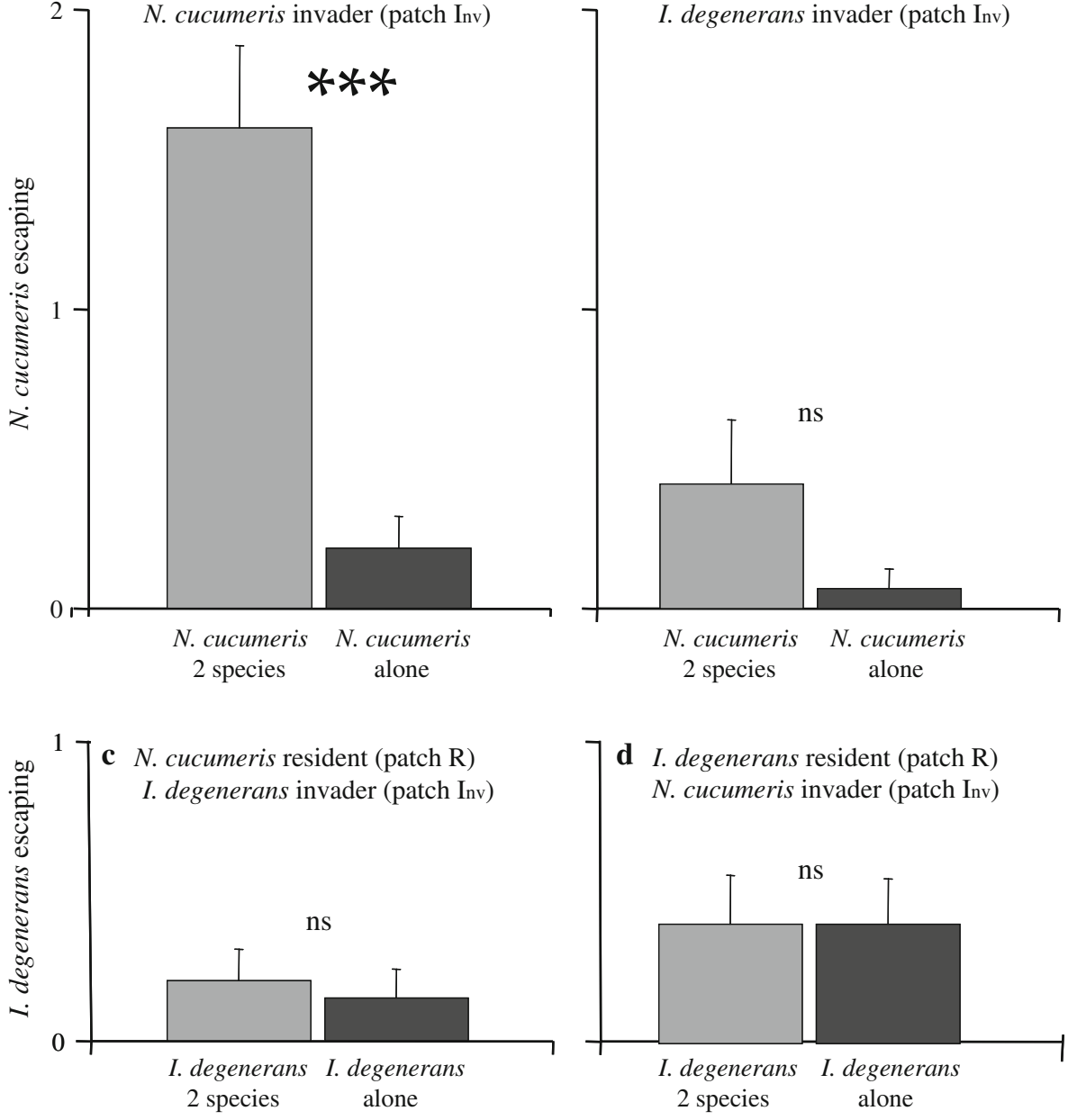

I. degenerans resident, $N$. cucumeris invading: response of I. degenerans

\section{Distribution through time (Fig. $3 g, h)$}

Distribution over patches with and without food: The proportion of I. degenerans on the pollen patches $(\mathrm{P}+\mathrm{R}$ vs. $\left.\mathrm{I}_{\mathrm{nv}}+\mathrm{E}\right)$ did not differ between the treatments with or without invading $N$. cucumeris (GLMM, binomial, $\chi_{1}^{2}=0.24$, $P=0.63)$. However, the distribution of I. degenerans through time differed in the presence of invading $N$. cucumeris (GLMM, binomial, treatment $\times$ time, $\left.\chi_{1}^{2}=8.01, P=0.005\right)$. The distribution of $I$. degenerans over the pollen patches and empty patches also changed through time (GLMM, binomial, time, $\chi_{1}^{2}=59.0$, $P<0.001)$. Thus, when $I$. degenerans was the resident species, its distribution over the empty patches and pollen patches changed over time and did so differently when $N$. cucumeris was invading.

Distribution over food patches: The distribution between the pollen patches ( $\mathrm{P}$ vs. R), also changed through time, depending on the presence of N. cucumeris (GLMM, bino- mial, treatment $\times$ time, $\quad \chi_{1}^{2}=5.66, \quad P=0.017$; time, $\left.\chi_{1}^{2}=98.9, \quad P<0.001\right)$. However, there was no significant effect of treatment (GLMM, binomial, treatment, $\left.\chi_{1}^{2}=0.42, P=0.52\right)$. Thus, through time there were differences in the distribution of I. degenerans between the two pollen patches, which depended on the presence of $N$. cucumeris, but the proportion on either patch did not differ in the presence of $N$. cucumeris.

Final distribution (Fig. 4d): The proportion of I. degenerans on the pollen patches after $24 \mathrm{~h}$ did not differ with the presence or absence of invading $N$. cucumeris, (GLM, binomial, $\mathrm{I}_{\mathrm{nv}}+\mathrm{E}$ vs. $\mathrm{P}+\mathrm{R}, \chi_{1}^{2}=1.58, P=0.21$ ). Neither was there a significant effect on the distribution over the patches with food (GLM, binomial, P vs. R, $\left.\chi_{1}^{2}=3.31, P=0.069\right)$, although there was a trend towards more $I$. degenerans residing on their patch of release $(\mathrm{R})$ in the presence of $N$. cucumeris.

Distribution of eggs (Fig. 5d): There was no significant effect of the presence of invading $N$. cucumeris on the distribution of eggs of I. degenerans (GLM, quasi-binomial, $\mathrm{I}_{\mathrm{nv}}+\mathrm{E} \quad$ vs. $\mathrm{P}+\mathrm{R}, \quad F_{1,28}=0.67, \quad P=0.42 ; \quad \mathrm{P} \quad$ vs. R, $\left.F_{1,28}=0.08, P=0.78\right)$. Thus, $I$. degenerans did not change 
oviposition site because of the presence of invading N. cucumeris.

Total number of eggs and escaped mites (Figs. 6d, 7d): In the presence of invading $N$. cucumeris, I. degenerans laid fewer eggs (GLM, Poisson, $\left.\chi_{1}^{2}=7.5, P=0.006\right)$. The number of mites that tried to escape was the same in both treatments $\left(F_{1,28}=0.0, P=1\right)$.

\section{Discussion}

Our results show that the behaviour of the reciprocal intraguild predators $N$. cucumeris and I. degenerans depended on the presence of the other species as well as on the order of invasion of the two species. When resident, neither species changed its distribution after $24 \mathrm{~h}$ in response to invasions of the other species. The only effect on the distribution of the resident species was when I. degenerans was resident-its distribution changed through time and this change was affected by the presence of $N$. cucumeris as invader. When invading, both species avoided patches with the other species, which confirms our hypothesis. However, there were also differences in the response of the two species; resident $N$. cucumeris hardly dispersed to the other food patch, whereas resident I. degenerans did. The lack of dispersal of resident $N$. cucumeris resulted in the other food patch being available to invading $I$. degenerans. In contrast, the dispersal of resident I. degenerans to the other food patch caused invading $N$. cucumeris to avoid both patches with food.

Juveniles are the most vulnerable to predation, and we therefore expected the distribution of eggs to change as a result of the presence of the other species. When it was the invader, $N$. cucumeris indeed deposited most of its eggs away from patches with high densities of I. degenerans. When it was the resident, $N$. cucumeris did not change the distribution of its eggs in the absence or presence of I. degenerans as the invader, and laid most eggs on the patch on which it had been released. This was expected, because most $N$. cucumeris adults remained on this patch, whereas I. degenerans avoided it.

I. degenerans did not change the distribution of its eggs in the presence or in the absence of $N$. cucumeris, irrespective of whether it was the resident or the invader. It did, however, seem to avoid ovipositing on both pollen patches when it was the invader, because the distribution of its eggs did not match the distribution of its adult females (cf. Figs. 4c, 5c). However, this was irrespective of the presence of $N$. cucumeris. We believe that I. degenerans may avoid ovipositing at patches with conspecifics to avoid cannibalism; apart from being an intraguild predator, I. degenerans also preys heavily on its own larvae and may therefore avoid ovipositing on places with conspecifics
(Ferreira et al. 2008). If I. degenerans indeed avoids ovipositing on patches with conspecifics to avoid cannibalism, avoidance should also have occurred when it was the resident. Indeed, the distribution of eggs also did not match the distribution of adults when I. degenerans was the resident, although a large proportion of eggs was oviposited on the patch of residence, on which many adults were present (cf. Figs. 4d, 5d).

I. degenerans females laid fewer eggs in the presence of $N$. cucumeris, regardless of whether they were the invader or the resident and $N$. cucumeris females laid fewer eggs when they were the invader. So even when food was not limiting, behavioural responses to each other's presence caused a decrease in oviposition rates, which would likely result in lower population growth rates. For I. degenerans, it is unclear what caused the decrease in oviposition. They had not moved from the pollen patches, thus did not seem to have refrained from feeding. Possibly, their mobility increased in the presence of $N$. cucumeris, causing them to spend less energy on oviposition. Alternatively, it could be that they postponed oviposition because they perceived the situation as risky. Such a behavioural response has been observed in N. cucumeris (Montserrat et al. 2007), and in I. degenerans (M. Montserrat, personal observation).

Invading $N$. cucumeris females reduced the time spent on the pollen patches in the presence of I. degenerans (Fig. 4a) and it is likely that this resulted in lower food consumption, causing a reduction in oviposition in $N$. cucumeris. Moreover, $N$. cucumeris tried to escape more often; perhaps they perceived the entire set-up as risky. In larger systems than the experimental arenas used here, they would possibly try to migrate further away to search for and occupy new patches with lower densities of predators.

This study examines only the short-term transient effects on the use of space. It remains to be investigated how the different distributions observed affect the dynamics of the two species. Theory predicts that reciprocal intraguild predators should not be able to invade populations of the other species (HilleRisLambers and Dieckmann 2003). We showed that invasion into populations of heterospecifics is actually avoided. This short-term avoidance may result in the aggregation of each species on separate patches, thus reducing the incidence of intraguild predation.

We demonstrated that the response of the predators depended on the context. Within our small experimental system, the behaviour of two predators differed depending on their order of introduction, with the effect that the ratios of the two species on the patches with food were unequal. In larger systems, with more food patches, this may lead to even more segregation between the species.

In conclusion, we suggest that studies on reciprocal intraguild predation should take into account that predators may adapt their behaviour, depending on subtle differences 
such as the order of invasion on a patch. This may result in partial segregation, which will weaken the interaction between intraguild predators and intraguild prey, which, in turn, is expected to increase the possibility of coexistence (May 1973; McCann et al. 1998; Arim and Marquet 2004).

Acknowledgments We thank Marta Montserrat, Belén Belliure, Roos van Maanen, Nicola Tiën, Ruixia Meng, Martijn Egas and Paulien de Bruijn for valuable discussions. Two anonymous reviewers are thanked for their comments, which improved the paper substantially. T. v. d. H. was employed by the University of Amsterdam within the framework of an ALW grant no 813.04.011 from the Netherlands Organization for Scientific Research (NWO).

Open Access This article is distributed under the terms of the Creative Commons Attribution Noncommercial License which permits any noncommercial use, distribution, and reproduction in any medium, provided the original author(s) and source are credited.

\section{References}

Agarwala BK, Yasuda H, Kajita Y (2003) Effect of conspecific and heterospecific feces on foraging and oviposition of two predatory ladybirds: role of fecal cues in predator avoidance. J Chem Ecol 29:357-376

Amarasekare P (2007) Trade-offs, temporal variation, and species coexistence in communities with intraguild predation. Ecology 88:2720-2728

Arim M, Marquet PA (2004) Intraguild predation: a widespread interaction related to species biology. Ecol Lett 7:557-564

Crawley MJ (2007) The R book. Wiley, West Sussex

Daugherty MP, Harmon JP, Briggs CJ (2007) Trophic supplements to intraguild predation. Oikos 116:662-677

Diehl S, Feissel M (2000) Effects of enrichment on three-level food chains with omnivory. Am Nat 155:200-218

Diehl S, Feissel M (2001) Intraguild prey suffer from enrichment of their resources: a microcosm experiment with ciliates. Ecology 82:2977-2983

Faraji F, Janssen A, Van Rijn PCJ, Sabelis MW (2000) Kin recognition by the predatory mite Iphiseius degenerans: discrimination among own, conspecific, and heterospecific eggs. Ecol Entomol 25:147-155

Faraji F, Janssen A, Sabelis MW (2001) Predatory mites avoid ovipositing near counterattacking prey. Exp Appl Acarol 25:613-623

Ferreira JAM, Eshuis B, Janssen A, Sabelis MW (2008) Domatia reduce larval cannibalism in predatory mites. Ecol Entomol 33:374-379

Finke DL, Denno RF (2002) Intraguild predation diminished in complex-structured vegetation: implications for prey suppression. Ecology 83:643-652

Finke DL, Denno RF (2006) Spatial refuge from intraguild predation: implications for prey suppression and trophic cascades. Oecologia 149:265-275

Heithaus MR (2001) Habitat selection by predators and prey in communities with asymmetrical intraguild predation. Oikos 92:542554

HilleRisLambers R, Dieckmann U (2003) Competition and predation in simple food webs: intermediately strong trade-offs maximize coexistence. Proc R Soc Lond B Biol Sci 270:2591-2598
Holt RD, Huxel GR (2007) Alternative prey and the dynamics of intraguild predation: theoretical perspectives. Ecology 88:2706-2712

Holt RD, Polis GA (1997) A theoretical framework for intraguild predation. Am Nat 149:745-764

Janssen A, Faraji F, van der Hammen T, Magalhaes S, Sabelis MW (2002) Interspecific infanticide deters predators. Ecol Lett 5:490 494

Janssen A, Montserrat M, HilleRisLambers R, De Roos AM, Pallini A, Sabelis MW (2006) Intraguild predation usually does not disrupt biological control. In: Brodeur J, Boivin G (eds) Guild interactions in biological control, vol 3. Springer, Dordrecht, pp 21-44

Janssen A, Sabelis MW, Magalhaes S, Montserrat M, Van der Hammen T (2007) Habitat structure affects intraguild predation. Ecology 88:2713-2719

Jolliffe PA (2000) The replacement series. J Ecol 88:371-385

Magalhaes S, Janssen A, Montserrat M, Sabelis MW (2005a) Prey attack and predators defend: counterattacking prey trigger parental care in predators. Proc R Soc B Biol Sci 272:1929-1933

Magalhaes S, Tudorache C, Montserrat M, van Maanen R, Sabelis MW, Janssen A (2005b) Diet of intraguild predators affects antipredator behavior in intraguild prey. Behav Ecol 16:364-370

May RM (1973) Stability and complexity in model ecosystems. Princeton University Press, Princeton

McCann K, Hastings A, Huxel GR (1998) Weak trophic interactions and the balance of nature. Nature 395:794-798

Montserrat M, Bas C, Magalhaes S, Sabelis MW, de Roos AM, Janssen A (2007) Predators induce egg retention in prey. Oecologia 150:699-705

Moran MD, Hurd LE (1994) Short-term responses to elevated predator densities-noncompetitive intraguild interactions and behavior. Oecologia 98:269-273

Morin P (1999) Productivity, intraguild predation, and population dynamics in experimental food webs. Ecology 80:752-760

Mylius SD, Klumpers K, de Roos AM, Persson L (2001) Impact of intraguild predation and stage structure on simple communities along a productivity gradient. Am Nat 158:259-276

Namba T, Tanabe K, Maeda N (2008) Omnivory and stability of food webs. Ecol Complex 5:73-85

Polis GA, Myers CA, Holt RD (1989) The ecology and evolution of intraguild predation-potential competitors that eat each other. Annu Rev Ecol Syst 20:297-330

R Development Core Team (2006) R Foundation for Statistical Computing, Vienna

Rosenheim JA, Kaya HK, Ehler LE, Marois JJ, Jaffee BA (1995) Intraguild predation among biological control agents-theory and evidence. Biol Control 5:303-335

Rudolf VHW (2007) The interaction of cannibalism and omnivory: consequences for community dynamics. Ecology 88:2697-2705

Sergio F, Marchesi L, Pedrini P (2003) Spatial refugia and the coexistence of a diurnal raptor with its intraguild owl predator. J Anim Ecol 72:232-245

Snyder RE, Borer ET, Chesson P (2005) Examining the relative importance of spatial and nonspatial coexistence mechanisms. Am Nat 166:E75-E94

Van Rijn PCJ, Tanigoshi LK (1999) Pollen as food for the predatory mites Iphiseius degenerans and Neoseiulus cucumeris (Acari: Phytoseiidae): dietary range and life history. Exp Appl Acarol 23:785-802

Venzon M, Janssen A, Pallini A, Sabelis MW (2000) Diet of a polyphagous arthropod predator affects refuge seeking of its thrips prey. Anim Behav 60:369-375 